\title{
Seeking precision in public health
}

\author{
'Precision' in a health context is usually thought to apply to the individual and seems conceptually at odds \\ with efforts in public health directed toward improving population-level health metrics. But are these two \\ aims truly irreconcilable?
}

T he tension between personalized medicine tailored for the individual and policies that best promote population health is particularly relevant in the age of big data and precision medicine, and in the context of the United Nations (UN) Sustainable Development Goals (https://sustainabledevelopment.un.org/sdgs). Increasingly, however, researchers and policymakers have begun seizing on the potential of 'precision public health', a controversial term with varied and evolving definitions. This September, as the global health agenda focuses on the High-Level Meeting on Universal Health Coverage as part of the 74th Session of the UN General Assembly, the question of how precise individualized metrics might inform publichealth policy should be carefully considered.

Today, increased data availability and new computational modeling methodologies allow for the mapping of health outcomes on a granular scale, thus enabling the identification of specific regions that might require additional support or implementation of targeted interventionsan approach known as spatial analysis. In this issue of Nature Medicine, Bhattacharjee et al. provide an example of fine-scale geospatial mapping in Africa and show how these data can inform policy at a regional level. Exclusive breastfeeding-providing only breastmilk with no additional food, water or milk-is one of the most effective strategies for improving infant nutrition, development and survival. Bhattacharjee et al. have produced data on a $5 \mathrm{~km} \times 5 \mathrm{~km}$ grid, estimating the prevalence of exclusive breastfeeding across 49 African countries from 2000 to 2017 . They show that $37 \%$ of infants in Africa were exclusively breastfed in the first 6 months of life, and only 18 countries in Africa are on track to achieve the World Health Organization (WHO) 50\% national Global Nutrition Target by 2025.

This fine-scale geospatial mapping powerfully illustrates how national statistics can mask regional heterogeneity and inequity. In South Africa, for example, where exclusive breastfeeding increased nationally by approximately $13.6 \%$ from 2000 to 2017 , in areas such as the City of Johannesburg and Gauteng province, increases have been much lower. These granular data identify the regions and communities that most need additional support, and can provide insights into specific causes underlying the disparities. In an accompanying News and Views, Reimers and colleagues discuss the barriers to exclusive breastfeeding in African countries. They highlight that educational interventions to support and encourage women to breastfeed must be regionally targeted as well as culturally tailored to the specific needs of communities.

Although the level of precision of these data will facilitate informed delivery of appropriate interventions at the local level, continuing accountability and surveillance at the national level remains crucial to meeting the WHO Global Nutrition Goals. The International Code of Breast-Milk Substitutes (the Code), for example, aims to restrict marketing and distribution of formula in ways that might interfere with promotion of breastfeeding. Although the Code was adopted by WHO member states in 1981, a 2016 report from the WHO and the UN Children's Fund indicated that only $30 \%$ of African countries had some form of legislation in place to support measures detailed in the Code.

Spatial epidemiology, although touted as a promising equitable approach to precision public health, is not the only existing strategy. In fact, an early example of precision in public health was a proposal from Julia Walsh and Kenneth Warren that a data-driven selective attack on the most severe public-health problems would be the most cost-effective strategy to reduce mortality and morbidity in limitedresourced settings (Walsh, J.A. and Warren, K.S. N. Engl. J. Med. 301, 967-974; 1979). This disruptive proposal to prioritize selected interventions appeared counter to the global movement toward promoting comprehensive primary health that followed the adoption of the Alma Ata Declaration in 1978. By proposing prioritization of selected interventions, Walsh and Warren defied the broad emphasis of the Declaration and triggered what is now an ongoing debate on universalism versus selective care.

A more contemporary, and perhaps the most controversial, interpretation of precision public health is the integration of big data (particularly genomics) into public-health decision-making processes. A number of high-profile projects, such as the UK 100,000 Genomes Project and the US National Institutes of Health's All of Us project (which aims to sequence 1 million Americans and analyze their health data), have been designed to improve diagnosis, treatment and care for individual patients and their families. However, the extent to which these precision-medicine approaches are relevant in the context of broader social, economic and structural drivers of population health has been hotly contested by experts (Chowkwanyun, M., Bayer, R. and Galea, S. N. Engl. J. Med. 379, 1398-1400; 2018). Challenges to the idea of integrating genomic data into publichealth policy are not only philosophical but also practical and ethical. The current lack of ethnic diversity in many large genomic datasets, for example, has the potential to widen health inequities by leaving traditionally understudied and underserved populations behind.

As the academic debate on defining what constitutes precision public health and how the concept aligns with a global health agenda geared toward universal health continues, a question lingers: is it time to eschew the nomenclature and to implement multidisciplinary approaches to the problem? Big data, fine-scale geospatial mapping, and appropriate prioritization of health interventions can each contribute in different ways to improving human health on a global scale. After all, health is health, by any other name.

Published online: 6 August 2019 https://doi.org/10.1038/s41591-019-0556-6 